\title{
APPROXIMATELY CONTINUOUS TRANSFORMATIONS ON COMPACT METRIC SPACES ${ }^{1}$
}

\author{
BY L. C. MARSHALL
}

Communicated by A. Zygmund, April 30, 1962

Goffman and Waterman [1] discussed approximately continuous transformations from the space $E_{n}$ to a metric space $T^{*}$. It was shown that the image, under such a transformation, of the space $E_{n}$ is a separable subset of $T^{*}$. Functions satisfying the Darboux property were discussed and it was shown that a function $f(x)$ defined on an interval $I$ possesses the Darboux property if and only if $f(x)$ takes connected sets of $I$ into connected sets. Homogeneous sets were introduced and it was shown that the class of all such sets of $E_{n}$ forms a topology called the $d$-topology. The concept of a $d$-regular set (a closed set with a connected interior) was introduced. Finally, it was shown that approximately continuous transformations take $d$ regular sets into connected sets, thus, generalizing the Darboux property.

Since the space $E_{n}$ is a metric space, it is natural to study such transformations in a more general setting. Accordingly, in this paper, we shall extend these matters to compact metric spaces.

1. Extension of the Darboux property to topological spaces. Let $p(t)$ be a single valued map of the unit interval into a topological space $T$. The set of points $C_{p}=E(p(t) \mid 0 \leqq t \leqq 1)$ is called a curve in the space $T$. The two points $p(0)$ and $p(1)$ are called the initial and terminal points, respectively, of the curve $C_{p}$. The curve is said to be closed if $p(0)=p(1)$. The set of points $C_{p}^{\prime}=E\left(p(t) \mid t_{1} \leqq t \leqq t_{2}\right)$ is called an arc of the curve $C_{p}$.

Let $f$ be a transformation of a connected space $T$ into itself and let $f_{r}$ be the restriction of $f$ to a curve $C_{p}$. Let $p\left(t_{1}\right)$ and $p\left(t_{2}\right)$ be the initial and terminal points, respectively, of an $\operatorname{arc} C_{p}^{\prime}$.

Definition. The transformation $f_{r}$ possesses the Darboux property if, for every arc $C_{p}^{\prime}$ of the curve $C_{p}$, the corresponding set of points $f_{r}\left(C_{p}^{\prime}\right)$ contains an arc whose initial and terminal points are $f_{r}\left(p\left(t_{1}\right)\right)$ and $f_{r}\left(p\left(t_{2}\right)\right)$, respectively.

Assume that $t_{1}$ different from $t_{2}$ implies that $p\left(t_{1}\right)$ is different from $p\left(t_{2}\right)$. Then the points $p(t)$ can be ordered by the relation $p\left(t_{1}\right)<p\left(t_{2}\right)$ if and only if $t_{1}<t_{2}$.

${ }^{1}$ This paper is a portion of a thesis, directed by Professor Elgy S. Johnson, to be submitted to The American University in partial fulfillment of the requirements for the degree of Doctor of Philosophy. 
It can be shown that the transformation $g(t)=f(p(t))$ is order preserving.

THEOREM 1. Let $f$ be a transformation of a topological space $T$ into itself and let $f_{r}$ be the restriction of $f$ to a curve $C_{p}$. Then $f_{r}$ possesses the Darboux property if and only if the image under $f_{r}$ of every connected subset of $C_{p}$ is also connected.

Proof. Assume that $f_{r}$ possesses the Darboux property and let $C_{p}^{\prime}$ be any arc of the curve $C_{p}$ such that the set $f_{r}\left(C_{p}^{\prime}\right)$ is not connected. There exist two points $g\left(\vec{t}_{1}\right)$ and $g\left(\vec{t}_{2}\right)$ which cannot be joined by an arc of a curve. Thus, there exists a real parameter $\tau: \bar{t}_{1}<\tau<\bar{t}_{2}$ such that the point $g(\tau)$ does not belong to the set $f_{r}\left(C_{p}^{\prime}\right)$. Let $p\left(\bar{t}_{1}\right)$ and $p\left(\bar{t}_{2}\right)$ be the inverse images of the points $g\left(\bar{t}_{1}\right)$ and $g\left(\vec{t}_{2}\right)$, respectively. Let $C_{p}^{\prime \prime}=E\left(p(t) \mid \vec{t}_{1} \leqq t \leqq \bar{t}_{2}\right)$ be an arc joining the two points $p\left(\bar{t}_{1}\right)$ and $p\left(\bar{t}_{2}\right)$. Since $f_{r}$ possesses the Darboux property, it follows that $f_{r}\left(C_{p}^{\prime \prime}\right)$ contains an arc whose end-points are $g\left(\bar{t}_{1}\right)$ and $g\left(\bar{t}_{2}\right)$, respectively. Furthermore, $\bar{t}_{1} \leqq \tau \leqq \bar{t}_{2}$, hence $g(\tau)$ belongs to the set $f_{r}\left(C_{p}^{\prime \prime}\right)$. This shows that $f_{r}\left(C_{p}^{\prime}\right)$ is, indeed, connected.

Assume now that the image of every connected subset of the curve $C_{p}$ is connected. Then $f_{r}$ possesses the Darboux property. Let $C_{p}^{\prime}$ be any connected subset of $C_{p}$ and let $p\left(t_{1}\right)$ and $p\left(t_{2}\right)$ be the initial and terminal points, respectively, of $C_{p}^{\prime}$. The set $f_{r}\left(C_{p}^{\prime}\right)$ is a connected set containing an arc whose end-points are $g\left(t_{1}\right)$ and $g\left(t_{2}\right) . C_{p}^{\prime}$ is arbitrary. Therefore, $f_{r}$ possesses the Darboux property. The theorem is proved.

The curve $C_{p}$ is said to be closed if $p(0)=p(1)$. The points $p(0)$ and $p(1)$ are called double points. We assume that the curve $C_{p}$ contains no other double points. We have the following

CoRollary. The transformation $f_{r}$ possesses the Darboux property if and only if $f_{r}$ takes closed sets into connected sets.

2. Approximately continuous transformations. Let $T$ be a compact space and denote by $S$ the $\sigma$-ring generated by the class of all compact subsets of $T$. A Borel measure is a measure $\mu$ defined on $S$ and such that $\mu(C)<\infty$ for every compact subset $C$. It is well known (1) that a regular content $\lambda$ can be extended to a regular Borel measure $\mu$ such that $\lambda(C)=\mu(C)$ for every compact set $C$. Therefore, $\mu$ is a non-negative, finite, monotone, additive and subadditive set function. These properties suffice to give a satisfactory account of approximately continuous transformations on compact metric spaces.

Definition. Let $S_{1}$ and $S_{2}$ be two Borel sets in the space $T$ and assume that the set $S_{2}$ is bounded. Then the relative measure of $S_{1}$ in $S_{2}$ is given by the number 


$$
M_{S_{1} S_{2}}=\mu\left(S_{1} \cap S_{2}\right) / \mu\left(S_{2}\right) .
$$

It can be shown that $0 \leqq M_{S_{1} S_{2}} \leqq 1$ for all pairs $S_{1}$ and $S_{2}$ such that $S_{2}$ is bounded. The upper and lower metric densities of $S_{1}$ at a point $p$ in the space $T$ are given by the numbers

$$
\begin{aligned}
& \phi^{*}(p)=\lim _{n \rightarrow \infty}\left(\operatorname{Sup}\left(M_{S_{1} S_{2}} \mid p \in S_{2} ; \mu\left(S_{2}\right)<\frac{1}{n}\right),\right. \\
& \phi_{*}(p)=\lim _{n \rightarrow \infty}\left(\operatorname{Inf}\left(M_{S_{1} S_{2}} \mid p \in S_{2} ; \mu\left(S_{2}\right)<\frac{1}{n}\right),\right.
\end{aligned}
$$

respectively. For all $p$ in the space $T$ we have the inequalities $0 \leqq \phi_{*}$ $\leqq \phi^{*} \leqq 1$. If $\phi_{*}=\phi^{*}$ we say that the metric density of $S_{2}$ exists at $p$ and equals the common value $\phi(p)$.

Definition. A transformation $f$ from the space $T$ to the space $T^{*}$ is approximately continuous at the point $p$ if, for every open set $G$ containing the point $f(p)$, the set $f^{-1}(G)$ has metric density one at $p$. The transformation is called approximately continuous if it is approximately continuous at each point of $T$.

THEOREM 2. The image of a compact space $T$ under an approximately continuous transformation is a separable subset of the metric space $T^{*}$.

Proof. Let $d$ and $d^{*}$ be the distance functions associated with the spaces $T$ and $T^{*}$, respectively. Let $\epsilon$ be any positive number and let $\left\{\epsilon_{i j}\right\}_{i, j=1}^{\infty}$ be a double sequence of positive numbers depending on $\epsilon$ in a manner to be specified later. Cover each point in the space with an open sphere each having its radius equal to $\rho_{0}$, where $\rho_{0}$ is a sufficiently small positive number. From the class of all such spheres we select a finite number, say $N_{0}$, which also covers the space $T$. The set of centers of these spheres $\left\{X_{0 i}\right\}_{i=1}^{N_{0}}$ determines a set of points $D=\left(f\left(x_{0 i}\right)\right) ; i=i, N_{0}$. Now, cover each point $f\left(x_{0 i}\right)$ with an open sphere $G_{0 i}$ of radius $\epsilon_{0 i}$. Since $f$ is approximately continuous at the point $x_{0 i}$ there exists a closed sphere $s_{0 i}$ such that the relative measure of the set $A_{0 i}=s_{0 i} \cap G_{0 i}^{-1}$ in $s_{0 i}$ exceeds $1-\epsilon_{0 i} / \mu\left(S_{0 i}\right)$. We may construct a finite sequence of disjoint sets $\bigcup_{i=1}^{N_{0}} z_{0 i}=\bigcup_{i=1}^{N_{0}} s_{0 i}$.

Let $E_{1}=\cup_{i=1}^{N_{0}} z_{0 i}$. The set $-T_{1}=\left(T-E_{1}\right)-$ is a closed subset of the compact space $T$ and is, therefore, compact. Thus, we may apply these same operations to the space $T_{1}$. Continuing in this manner we obtain a sequence of pairwise disjoint sets $E_{k}$ giving rise to a corresponding sequence of subspaces and associated sets

$$
D^{(k)}=U\left\{f\left(X_{k i}\right)\right\}_{i=1}^{N_{k}} \text {. }
$$


It can be shown that (1) $T=\bigcup_{j=1}^{\infty} E_{j}$ and (2) $D=\bigcup_{j=1}^{\infty} D^{(j)}$ is denumerable. Let $s_{k i}$ be the sphere with center at $x_{k i}$ and let $G_{k i}^{-1}$ be the associated set defined above. It has been shown that the relative measure of the set $A_{k i}$ in $s_{k i}$ exceeds $1-\epsilon_{k i} / \mu\left(S_{k i}\right)$.

Lemma 1. Let $A_{k i}^{*}=s_{k i}-A_{k i}$. Then $\mu\left(A_{k i}^{*}\right)<\epsilon_{k i}$.

Proof. $\mu\left(s_{k i}\right)=\mu\left(A_{k i} \cup A_{k i}^{*}\right)=\mu\left(A_{k i}^{*}\right)+\mu\left(A_{k i}\right)$. Therefore,

$$
\mu\left(A_{k i}^{*}\right) / \mu\left(s_{k i}\right)=1-\mu\left(A_{k i}\right) / \mu\left(s_{k i}\right) .
$$

But,

$$
\mu\left(A_{k i}\right) / \mu\left(s_{k i}\right)>1-\epsilon_{k i} / \mu\left(S_{k i}\right) .
$$

Therefore,

$$
-\mu\left(A_{k i}\right) / \mu\left(s_{k i}\right)<-1+\epsilon_{k i} / \mu\left(S_{k i}\right) .
$$

Hence,

$$
\mu\left(A_{k i}^{*}\right)<\left(\epsilon_{k i}\right)\left(\mu\left(s_{k i}\right) / \mu\left(S_{k i}\right)\right) .
$$

Since $s_{k i} \subseteq S_{k i}$ it follows that $\mu\left(A_{k i}^{*}\right)<\epsilon_{k i}$.

Lemma 2. Let $\epsilon$ be any positive number and let $\Lambda$ be the set of points of $T$ for which $d^{*}(f(\lambda), D)>\epsilon$ for every $\lambda \in \Lambda$. Then $\mu(\Lambda)<\epsilon$.

PROOF. It has been shown that $T=\bigcup_{k=1}^{\infty} E_{k}=\bigcup_{j=1}^{\infty}\left\{\cup_{i-1}^{N_{i}} s_{i j}\right\}$. Furthermore, it can be shown, without difficulty, that

$$
\Lambda=\bigcup_{i=1}^{\infty}\left\{\bigcup_{j=1}^{N_{i}} A_{i j}^{*}\right\} \text {. }
$$

Lemma 1 shows that $\mu\left(A_{k i}^{*}\right)<\epsilon_{i j}$. Therefore,

$$
\mu(\Lambda)=\mu\left(\bigcup_{i=1}^{\infty}\left(\bigcup_{j=1}^{N_{i}} A_{k i}^{*}\right)\right) \leqq \sum_{i=1}^{\infty}\left\{\sum_{j=1}^{N_{i}} \epsilon_{i j}\right\} .
$$

Let

$$
\epsilon_{i j}=\frac{1}{2} \frac{\epsilon}{2^{N_{0}+N_{1}+\cdots+N_{i-1}+j}} .
$$

It follows that

$$
\mu(\Lambda) \leqq \frac{1}{2} \epsilon \sum_{\nu=1}^{\infty} \frac{1}{2^{\nu}}<\epsilon .
$$

The theorem can now be demonstrated. Clearly, the set $D$ is dense in $f(T-\Lambda)$. Let $\lambda$ be any point in the set $\Lambda$ and let $G$ be any open 
sphere about $f(\lambda)$. Since $f$ is approximately continuous at $\lambda$ there exists a point $t$ in the space $T$ such that $d^{*}(f(t), f(\lambda))<\delta / 2$. There exists a point $\zeta$ in $D$ such that $d^{*}(f(t), \zeta)<\delta / 2$. Therefore, $d^{*}(f(\lambda), \zeta)$ $\leqq d^{*}(\zeta, f(t))+d^{*}(f(\lambda), f(t))<\delta$. This shows that $D$ is also dense in the set $f(T)$, and the theorem is proved.

3. Homogeneous sets and the $d$-topology. The concept of a homogeneous set can readily be extended to compact metric spaces. A Borel set $S$ is said to be homogeneous if its metric density is one at each of its points. The class of all such sets forms a topology referred to as the $d$-topology. The concepts of $d$-limit points and $d$-connected sets are extended in exactly the same way. The proofs that this topology is Hausdorff and that the class of approximately continuous transformations coincides with the class of all continuous transformations can also be extended without major changes. Furthermore, it is not difficult to prove that approximately continuous transformations take $d$-connected sets into connected sets.

Assume that our compact metric space $T$ is arc-wise connected and that the sequence $d_{n}=d\left(p\left(t_{n}\right), p\left(t_{0}\right)\right)$ approaches zero for every sequence of points $p\left(t_{n}\right)$ approaching the point $p\left(t_{0}\right)$.

LeMma 1. For every Borel set $A$ in the space $T$, the relative measure of the set in the sphere $S_{p r}$ is a continuous function of $p$ for fixed $r$.

Proof. Let $p_{0}$ be an arbitrary but fixed point and let $p_{n}$ be any point different from $p_{0}$. Let $C_{p}$ be a curve joining these points. Let $S_{p_{0} r}$ and $S_{p_{n} r}$ denote spheres with centers at $p_{0}$ and $p_{n}$, respectively. Define $F_{n}=S_{p_{0} r}-S_{p_{n} r}$. We assert that $F_{n}$ is a monotone decreasing sequence whose limit is the null set. Let $S=\mathrm{U}_{p \in C_{p}} S_{p r}$. Clearly, the class of open spheres $S_{p_{n} r}$ is a subset of $S$. Let $Q$ be any point in $S$ for which $d^{*}\left(p\left(t_{0}\right), Q\right)>r$. It suffices to show that there exists a positive integer $N$ such that $d\left(p\left(t_{0}\right), Q^{\prime}\right) \leqq d\left(p\left(t_{0}\right), Q\right)$, for every point $Q^{\prime}$ belonging to the sphere $S_{p_{n} r}(n \geqq N)$. Indeed, let $d\left(p\left(t_{0}\right), Q\right)=r+\beta(\beta>0)$. Since $p\left(t_{n}\right)$ approaches $p\left(t_{0}\right)$ there exists a positive integer $N(\beta)$ such that $d\left(p\left(t_{0}\right), p\left(t_{n}\right)\right)<\beta / 2$, for all $n \geqq N(\beta)$. Let $Q^{\prime}$ be any point in $S_{p_{n} r}(n \geqq N(\beta))$. Then $d\left(p\left(t_{0}\right), \quad Q^{\prime}\right) \leqq d\left(p\left(t_{0}\right), \quad p\left(t_{n}\right)\right)+d\left(p\left(t_{n}\right), \quad Q^{\prime}\right)$ $<\beta / 2+r<d\left(p\left(t_{0}\right), Q\right)$. Since $Q$ is arbitrary it follows that $F_{n} \rightarrow 0$. Therefore, $A \cap S_{p_{n} r} \rightarrow A \cap S_{p_{n} r}$. Hence $\mu\left(A \cap S_{p_{n} r}\right) \rightarrow \mu\left(A \cap S_{p_{0} r}\right)$. This proves the lemma.

Theorem 3. Every open connected subset of the space $T$ is $d$-connected.

Proof. Having proved Lemma 1, Theorem 3 [1] can be extended 
almost word for word to the present case if we replace the concept of an oriented closed cube by an open sphere.

4. $d$-regular sets. A closed set (with connected interior) whose boundary points are $d$-limit points of the interior is called $d$-regular. Theorem 3 leads directly to

ThEOREM 4. Every d-regular set is d-connected.

Theorem 4 leads to a generalization of the Darboux property. Indeed, a remarkable relationship between the Darboux property and approximately continuous transformations is established by

THEOREM 5. Approximately continuous transformations take d-regular sets into connected sets.

PROOF. Theorem 4 shows that $d$-regular sets are $d$-connected. We have remarked that approximately continuous transformations take $d$-connected sets into connected sets.

\section{REFERENCES}

1. C. Goffman and D. Waterman, Approximately continuous transformations, Proc. Amer. Math. Soc. 12 (1961), 116-121.

2. P. Halmos, Measure theory, Van Nostrand, New York, 1950.

The American University and

Republic Aviation Corporation 